\title{
Volume Administered
}

National Cancer Institute

\section{Source}

National Cancer Institute. Volume Administered. NCI Thesaurus. Code C69279.

The amount of substance given a subject. 